\title{
An Approach to Understand Semantic Web
}

\author{
Deepti Juneja \\ Research Scholar \\ School of Engineering and Technology \\ Jagannath University, Jaipur
}

\author{
M. K. Sharma, PhD \\ Associate Professor \\ Department of Computer Applications \\ Amrapali Institure, Haldwani
}

\begin{abstract}
The current internet relies around markup language pages delivered to a Browser and afterward displayed for human consumption instead of for important manipulation by $\mathrm{pc}$ applications. Holzschlag (2002) states the first goal of markup language was to produce document markup for files sent via the web and, at heart, it's merely a document terminology specializing in the structure of a document. The present HTML-based World Wide internet was originally formed and created by Tim Berners-Lee at CERN, the European organization for physics analysis, to permit scientists UN agency frequently changed papers to reference alternative papers. The Semantic Web represents his vision for consecutive generation internet of the long run (Updegrove, 2005).
\end{abstract}

\section{General Terms}

Semantic Web, Semantic Web Framework, Data Modeling, Semantic Web Stack

\section{Keywords}

Layer Cake Architecture, Modeling Information, RDF, Web Ontology Language

\section{INTRODUCTION}

"The Semantic Web is not a different Web but rather an expansion of the present one, in which data is given very much defined importance, better empowering PCs and individuals to work in collaboration." [Tim Berners-Lee]. The Semantic Web has been depicted as a dream of a cutting edge Web that gives distributers a chance to make pages containing documentations intended to give "signifying" to the information inside the page content [Ogbuji, 2002]. Semantics just means meaning. It gives a word or image valuable importance through the foundation of connections.

The semantic web is just a web of information depicted and connected in approaches to build up setting or semantics that hold fast to characterized syntax and dialect develops. The Semantic Web is best comprehended in contrast with the World Wide Web (WWW). Table 1 [1] thinks about the two. As opposed to being a substitute for the WWW, the Semantic Web broadens it through useable, institutionalized semantics that draw profoundly on scholastic research in learning portrayal and rationale to approach the objective of universal mechanized data sharing.
Table 1. Comparison of WWW and SW

\begin{tabular}{|c|c|c|}
\hline Feature & WWW & Semantic Web \\
\hline $\begin{array}{c}\text { Fundamental } \\
\text { component }\end{array}$ & Unstructured content & $\begin{array}{c}\text { Formal } \\
\text { Statements }\end{array}$ \\
\hline $\begin{array}{c}\text { Primary } \\
\text { audience }\end{array}$ & Humans & Applications \\
\hline Links & Indicate location & $\begin{array}{c}\text { Indicate location } \\
\text { and meaning }\end{array}$ \\
\hline Vocabulary & $\begin{array}{c}\text { Formatting } \\
\text { instructions }\end{array}$ & $\begin{array}{c}\text { Semantics and } \\
\text { logic }\end{array}$ \\
\hline Logic & Informal/Nonstandard & Description logic \\
\hline
\end{tabular}

The WWW comprises fundamentally of substance for human utilization. Content connections to other substance on the WWW by means of the Universal Resource Locator (URL). The URL depends on encompassing setting (ifany) to impart the motivation behind the connection that it speaks to; generally the client deduces the semantics. Web content regularly contains designing guidelines for a decent introduction, again for human utilization. WWW content does not have any formal intelligent builds. Correspondingly, the Semantic Web comprises essentially of articulations for application utilization. The announcements interface together through develops that can frame semantics, the significance of the connection. Consequently, connect semantics give a defined significant way as opposed to a client deciphered one. The announcements may likewise contain rationale that permits facilitate understanding and derivation of the announcements.

\section{LAYER CAKE ARCHITECTURE OF SEMANTIC WEB}

The Semantic Web is only an expansion of the present one, wherein data is given all around characterized importance, and help PCs and individuals to work in participation better [1]. The Semantic web distinguishes an arrangement of innovations, apparatuses, and benchmarks which frames the essential building squares of a foundation to bolster the vision of a web with significance. The Semantic web design comprises of a progression of principles composed into a layered structure that communicates their interrelationships. The engineering of the Semantic Web comprises of chain of importance of dialects and advancements, every dialect both misusing the elements and expanding the capacities of the layers beneath. This has been represented in Tim BernersLee's "Semantic Web Stack" outline [2]. The Semantic Web Stack, otherwise called Semantic Web Cake or Semantic Web Layer Cake, demonstrates the engineering of the Semantic 
Web as appeared in Fig. 1 [2]. It demonstrates how advances that are institutionalized by W3C for Semantic Web are sorted out to make the Semantic Web conceivable. It additionally demonstrates how Semantic Web is an expansion of the established hypertext web.

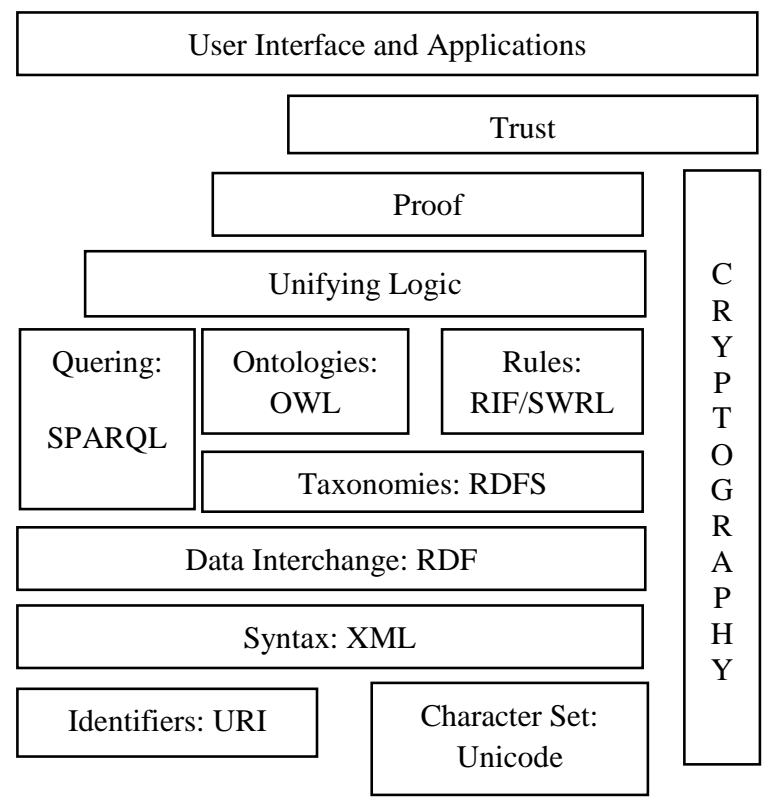

Fig 1: The Sematic Web Stack

- URI: A Uniform Resource Identifier gives a one of a kind name to things contained in an announcement over the whole Internet. Along these lines, every segment of an announcement-subject, predicate, and protest - contains a URI to affirm its personality all through the whole WWW. This disposes of naming conflicts, guarantees that two things are the same or not, and can likewise give a way to extra data.

- $\quad$ eXtensible Markup Language (XML): It is a dialect for information trade on web permitting the organizing of information however without imparting the importance of the information. XML and its related gauges, for example, XML Namespaces, and XML Schemas, shape a typical means for organizing information on the Web are as of now entrenched inside the Web.

- Resource Description Framework (RDF): RDF is the primary layer of the Semantic Web. It depicts the assets on the web. It is a basic metadata portrayal structure that utilizations URIs to recognize Web-based assets and a diagram display for depicting connections between these assets. A few syntactic portrayals are accessible, including a standard XML arrange. It is a dialect for institutionalizing the definition and utilization of metadata for data portrayal on the web.

- $\quad$ RDF Schema (RDFS): It is a straightforward sort demonstrating dialect for portraying and making orders of classes of assets and properties between them in the essential RDF display. It additionally gives a basic thinking system to construing sorts of assets. It gives fundamental vocabulary to RDF.
- Ontology: An ontology consists of statements that define concepts, relationships, and constraints. It is comparable to a database composition or a question situated class graph. The ontology forms an information domain model.

- Logic and Proof: It is a (programmed) thinking framework set on top of the ontology structure to make new derivations. Consequently, utilizing such a framework, a product operator can make findings with respect to whether a specific asset fulfills its necessities.

- $\quad$ Rules: This layer offers help for guidelines. This is vital to permit portraying relations that can't be straightforwardly depicted, for instance utilizing Description Logic (DL) utilized as a part of Web Ontology Language (OWL).

- Cryptography: To guarantee and confirm that semantic web explanations are originating from put stock in source, fitting advanced mark of RDF proclamations must be appended.

- Trust: It addresses the issues of trust that the Semantic Web can bolster. The vision is of enabling individuals to make inquiries of the reliability of the data, for giving an affirmation of its quality. Trust of the inferred explanations will be upheld by (a) checking that the premises originated from put stock in source and by (b) depending on formal rationale amid determining new data.

\section{MODELING INFORMATION}

In computer code systems, data modeling is of the utmost importance. The attributes of real-world objects that you just opt to capture in your computer code and therefore the means that you just opt to represent them for the most part confirm the operations that your system will perform and therefore the queries that it will answer. In a semantic web this is often even additional necessary, as a result of by modeling ideas and objects with sufficient rigor, it's attainable to apply information from one application in another. On the semantic web, data is shapely primarily with a collection of 3 complementary languages: the Resource Description Framework (RDF), RDF Schema (RDFS), and Web Ontology Language (OWL). RDF defines the underlying information model and provides a foundation for the additional refined options of the upper levels of the Semantic Web layer cake.

\subsection{The Semantic web Information Model: The RDF}

On the Semantic Web, data is described as a collection of assertions known as statements created from 3 parts: subject, predicate, and object. Owing to these 3 components, statements also are typically said as triples. The 3 components of a statement have meanings that are analogous to their meanings in traditional English grammar. The subject of a statement is that the factor that statement describes, and therefore the predicate describes a relationship between the subject and therefore the object. To clarify, think about the subsequent listing: 
Peter knows Mathew.

Peter's surname is William.

Mathew knows James.

Robert works with James

\section{Listing 1}

Fig. 2 is a graphical illustration of that tiny set of knowledge. Assertions of this kind naturally form a directed graph, with subjects and objects of every statement as nodes, and predicates as edges. This is often the information model employed by the semantic net, and it's formalized within the language known as the Resource Description Framework (RDF).

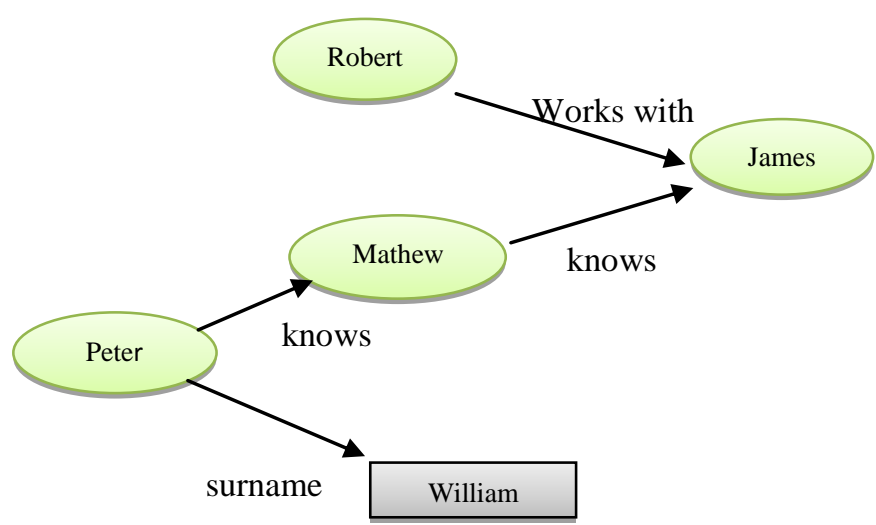

Fig 2: A graph representation of the sentences

\section{SEMANTIC WEB FRAMEWORKS}

Most Semantic Web frameworks are a group of integrated tools that enable you to make and work with a knowledgebase. The framework is that the set of tools; the knowledgebase is that the capability or idea of what they succeed. These frameworks are typically composed of 3 basic forms of elements, as represented in Figure 3: storage, access, and inference. [1] Each element is interconnected as a result of theirs typically lots of interaction among these numerous elements.

- Storage elements are repositories of RDF statements that store information.

- Access elements are typically question processors or application programming interfaces (APIs) that offer the retrieval and modification of knowledge, and

- Inference elements are reasoning engines that apply interpretation of OWL semantics to the knowledge within the knowledgebase.

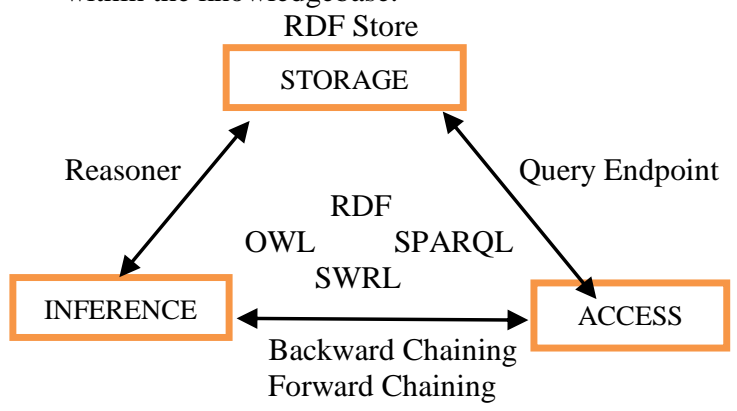

Fig 3: A Semantic Web Framework
Fundamentally, a knowledgebase may be an assortment of facts (statements). The elements of a semantic net framework serve to store, offer access to, and infer regarding these facts. Facts will be specific or implicit. Specific facts are those who are directly declared within the knowledgebase. Implicit facts are entailments, facts whose existence is understood by the mix of specific facts and also the linguistics of ontologies and rules within the knowledgebase. Entailments are derived by the reasoning element of the knowledgebase. Counting on implementation, entailments could also be hold on directly within the underlying storage mechanism or they'll be derived as required once data is retrieved from the knowledgebase. Content implementations could perform logical thinking mechanically or not, and that they will perform logical thinking internally among the knowledgebase or through an external process.

\section{CONCLUSION}

The semantic net provides opportunities for users to get higher search results and acquire additional targeted traffic as users notice what they actually need just in case of site house owners. However these edges don't simply as if by magic appear. Though the aim of Tim Berners-Lee's Semantic internet is nonetheless to be totally complete, the years of thinking and analysis that have gone into it are starting to bear fruit in terms of solutions to practical issues that individuals face nowadays. The strong collaboration trends in internet can solely lead to additional necessities for structured and semantically encoded knowledge being accessible on the Web.

In spite of many efforts by researchers and developers, SW has remained a future concept or technology. It is not practiced presently because Complete Semantic Web (CSW) has not been developed yet and no optimal software or hardware is provided. This paper gives a brief about the overview of semantic web, the modeling information, its architecture and framework. We know that SW can prove a boon for Information Retrieval and Pattern Recognition concepts, but for that CSW has to be practically implemented in the real world.

\section{ACKNOWLEDGEMENT}

I want to thank my supervisor Dr. M. K. Sharma for all his time, support and feedback. He has been a professional support for guiding the research and responsible for creating an exuberant and inspiring research environment.

\section{REFERENCES}

[1] John_Hebeler. Semantic Web programming. Available at Wiley Publishing.

[2] Tim Berners-Lee (1999) Weaving the Web. Available at http://www.w3.org/People/ Berners-Lee/Weaving

[3] Semanticweb.org (2009) The Semantic Web. Available at http://semanticweb.org/wiki/ Main_Page

[4] W3C. W3C Semantic Web Activity. Available at http://www.w3.org/2001/sw/

[5] The Semantic Web: An Introduction. Available at http://infomesh.net/2001/swintro/

[6] N.Shadbolt, T.Berners Lee and W. Hall, "The Semantic Web revisited",'IEEE Intelligent Systems” 2006.

[7] Berners-Lee and Fischetti, "Weaving the Web: The Original Design of the World Wide Web by its inventor", 
"Scientific American", 2005.

[8] Kiryakov, A. Popov, L. Manov, "Semantic annotation, indexing and retrieval", "Journal of Web Semantics", 2005-2006.

[9] J. Kopena, A.Joshi, "DAMLJessKB: A tool for reasoning with Semantic Web”, "IEEE Intelligent Systems”, 2006.
[10] Zou, Y.Y., Finin, T. and Chen, H. (2004) F-OWL: An Inference Engine for the Semantic Web. Formal Approaches to Agent-Based Systems. Vol. 3228 of Lecture Notes in Computer Science. Springer-Verlag, Berlin. Proceedings of the Third International Workshop (FAABS), 16-18 April 2004. 